\title{
Spectroscopic binaries with elliptical orbits
}

\author{
L. B. Lucy \\ Astrophysics Group, Blackett Laboratory, Imperial College London, Prince Consort Road, London SW7 2AZ, UK \\ e-mail: 1.lucy@imperial.ac.uk \\ Received 21 February 2005 / Accepted 8 May 2005

\begin{abstract}
The radial velocity curves of many spectroscopic binaries (SBs) are perturbed by gas streams or proximity effects. For SBs with circular orbits, these perturbations can give rise to spurious orbital eccentricities of high statistical significance. But tests to identify such anomalous orbits can be constructed since perturbed velocity curves are in general no longer Keplerian. The derived tests are applied both to synthetic and to observed velocity curves.
\end{abstract}

Key words. binaries: spectroscopic - methods: statistical

\section{Introduction}

Observers of spectroscopic binaries and exoplanets commonly test the statistical significance of orbital eccentricities if $e \lesssim$ 0.1. The widely adopted methodology (Lucy \& Sweeney 1971 , 1973 ) is to test the null hypothesis $H_{0}$ that $e=0$. If $H_{0}$ is rejected at the 5 per cent level, the elliptical orbit is accepted. If not, the circular orbit is preferred.

Although recommending and implementing this methodology, Lucy \& Sweeney (1971, hereafter LS) noted that some $e$ 's thus accepted are spurious, being due to absorption line distortions by gas streams or proximity effects. Accordingly, they suggested that, if $e>0$ is essential for a subsequent investigation, then the 1 per cent level of significance could be adopted. Nevertheless, they noted that the well known shortperiod eclipsing binary U Cephei, for which photometric evidence indicates $e=0$, is still assigned an elliptical orbit at the 1 and even the 0.1 per cent level of significance.

Given that changing the level of significance in the LS-test is not a secure method of identifying a sample of SBs free from spurious $e$ 's, a different approach is required to detect perturbations. The expectation that a perturbed velocity curve will in general not be strictly Keplerian is the basis for the additional tests developed in this paper.

This investigation is prompted by the work of Skuljan et al. (2004). Exploiting the high-precision radial velocities attainable with a modern spectrograph, they report new orbital elements for the single-lined SB $\zeta$ TrA derived from measurements with standard error $\sigma=0.014 \mathrm{~km} \mathrm{~s}^{-1}$, a vast improvement on the previous orbit by Spencer Jones (1928) for which $\sigma=1.4 \mathrm{~km} \mathrm{~s}^{-1}-$ see entry No. 445 in Table 1 of LS. With this jump in precision, it is no great surprise that a statistically insignificant $e$ of 0.06 is now replaced by a highly significant value of $0.0140 \pm 0.0002$. Moreover, the other elements are correspondingly improved in precision. In particular, $\sigma_{\omega}$, the error on the longitude of periastron $\omega$ is reduced from $63^{\circ}$ to a mere 0.9

With this remarkable reduction in $\sigma_{\omega}$, the time interval required for the detection of apsidal motion for this and similar SBs is greatly reduced, raising the prospect of greatly increasing the data set for this classic test of stellar structure. But before making second epoch observations, an investigator should check that $e$, no matter how significant according to the LS-test, passes the further tests developed in this paper.

\section{Harmonic analysis}

Nowadays, orbital elements are almost exclusively determined by least-squares fitting; and the LS-test as well as the tests developed herein are specific to such solutions. Nevertheless, these new tests are more readily understood in the context of determining the elements from a harmonic analysis of the velocity curve (Wilsing 1893; Russell 1902; Monet 1979).

\subsection{Fourier expansion}

The Keplerian velocity $V$ of a component of an SB with orbital period $P$ is

$V=\gamma+K[\cos (\nu+\omega)+e \cos \omega]$

where $\gamma$ is the radial velocity of the barycentre, $K$ is the semiamplitude, and $v(t)$ is the true anomaly. Since the motion is periodic, $V(t)$ can be expanded as a Fourier series,

$V=\frac{1}{2} C_{0}+\sum_{j=1}^{\infty}\left[C_{j} \cos j L+S_{j} \sin j L\right]$

where $L=2 \pi\left(t-T_{0}\right) / P$ is the mean longitude.

For given orbital elements $P, T_{0}, \gamma, K, e \cos \omega, e \sin \omega$, the Fourier coefficients $C_{j}$ and $S_{j}$ are derivable from Eq. (1) by 
numerical integration of the Fourier integrals. Alternatively, for $e \lesssim 0.5$, accurate analytic formulae can be obtained by expanding in powers of $e$. Such formulae are given in the Appendix for the coefficients of the first four harmonics.

\subsection{Estimating orbital elements}

Given numerous measurements of a component's radial velocity, the orbital elements may be estimated as follows. First, $P$ is determined by minimizing the scatter about the mean velocity curve. Then, with a trial value of $T_{0}$, the coefficients $C_{j}$ and $S_{j}$ are estimated by approximating their Fourier integrals by summations over the observed velocities. The final step is to derive the orbital elements algebraically by equating these estimates, $\tilde{C}_{j}$ and $\tilde{S}_{j}$, to their Keplerian counterparts.

With only the leading term retained from the expressions in Appendix A, we have $C_{0}=2 \gamma, C_{1}=K, S_{1}=0, C_{2}=$ $K e \cos \omega$, and $S_{2}=K e \sin \omega$. Accordingly, if $T_{0}$ is adjusted so that $\tilde{S}_{1}=0$, then $\gamma=\tilde{C}_{0} / 2, K=\tilde{C}_{1}, e \cos \omega=\tilde{C}_{2} / K$, and $e \sin \omega=\tilde{S}_{2} / K$. These elements can be improved iteratively to allow for higher order terms in $e$ in the formulae for $C_{1,2}$ and $S_{1,2}$.

\subsection{Perturbed velocity curves}

A perturbation due to gas streams or proximity effects will result in estimates $\tilde{C}_{j}$ and $\tilde{S}_{j}$ that differ systematically from their exact Keplerian values. But such differences in $\tilde{C}_{1,2}$ and $\tilde{S}_{1,2}$ do not cause the above solution procedure to fail, and so the investigator remains unaware both of the perturbation and of the resulting systematic errors in the elements.

To detect a perturbation, the analysis must be extended to the third harmonic, for which the leading terms are $C_{3}=$ $9 / 8 K e^{2} \cos 2 \omega$ and $S_{3}=9 / 8 K e^{2} \sin 2 \omega$. Evidently, $C_{3}$ and $S_{3}$ are predicted by the complete set of elements derived above from $\tilde{C}_{1,2}$ and $\tilde{S}_{1,2}$, leaving no adjustable parameters to fit $\tilde{C}_{3}$ and $\tilde{S}_{3}$. Accordingly, if the velocity curve is perturbed, the vector $\left(\tilde{C}_{3}, \tilde{S}_{3}\right)$ will in general differ from the prediction $\left(C_{3}, S_{3}\right)$. This is the basis for the test developed in Sect. 3.2.

\section{A hierarchy of tests}

In this section, tests are developed for separating SBs with perturbed velocity curves from those consistent with pure Keplerian motion. Each test compares the goodness-of-fits for a pair of models, where one of the pair is derivable from the other by imposing constraints on its parameters.

The goodness-of-fit criterion for model $k$ is the sum of weighted squared residuals

$R_{k}=\sum_{n} w_{n}\left(v_{n}-V_{n}\right)^{2}$

where $v_{n}$ is the observed radial velocity with relative weight $w_{n}$, and $V_{n}$ is the predicted velocity.

This sequence of tests starts with the LS-test for statistically significant $e$ 's. For completeness and clarity, this test is briefly recapitulated here.

\subsection{Detection of ellipticity}

Given the data set $\left(v_{n}, w_{n}, t_{n}\right)$, we first calculate the leastsquares circular orbit $(k=1)$, thus determining the elements $P, T_{0}, \gamma$ and $K$. Next, the least-squares elliptical orbit $(k=2)$ is computed, thus determining the elements $P, T_{0}, \gamma, K, e \cos \omega, e \sin \omega$. Because of the two additional parameters, the elliptical orbit is always a better fit to the data, so that $R_{2}<R_{1}$. The question is whether the reduction justifies rejecting the circular orbit. To assess this, LS treated the problem as one of testing multivariate linear hypotheses. In this case, the null hypothesis of a circular orbit is obtained by adopting the following linear hypotheses

$e \cos \omega=0, \quad e \sin \omega=0$

with respect to two of the orbital elements.

Applying standard statistical theory, LS found that the probability that measurement errors of an SB with $e=0$ could result in a least-squares elliptical orbit with $R \leq R_{2}$ is

$p_{1}=\left(\frac{R_{2}}{R_{1}}\right)^{\beta_{2}}$.

Here $\beta_{2}=1 / 2\left(N-M_{2}\right)$, where $N$ is the number of measured velocities, and $M_{2}$ is the number of elements for the elliptical orbit - i.e., for the unconstrained model.

The probability $p_{1}$ determines the outcome of the first decision we make about this SB: the circular orbit is accepted if $p_{1}>0.05$, and the elliptical orbit if $p_{1}<0.05$.

If the $v_{n}$ were subject only to measurement errors, this decision would conclude the investigation. Thus, if $p_{1}<0.05$, the reality of $e$ would be accepted. But now we question the reality of such statistically significant $e$ 's.

\subsection{Detection of perturbation}

In Sect. 2.3, we concluded that a perturbation will in general cause a displacement between the vectors $\left(\tilde{C}_{3}, \tilde{S}_{3}\right)$ and $\left(C_{3}, S_{3}\right)$. In the context of a least-squares solution, this implies that $v_{n}-$ $V_{n}$, the residuals remaining after fitting an elliptical orbit, will still contain a 3rd harmonic component.

To look for this, consider the following two-parameter model

$V=V_{\mathrm{ell}}+\Delta C_{j} \cos j L+\Delta S_{j} \sin j L$.

Here $V_{\text {ell }}$ is the previously-determined elliptical orbit, and the additional terms are an adjustable $j$ th harmonic, with $j=3$ unless otherwise specified. The least-squares solution for this model $(k=3)$ determines the vector $\Delta_{j}=\left(\Delta C_{j}, \Delta S_{j}\right)$, with the best-fit subsequently written as $\delta_{j}=\left(\delta C_{j}, \delta S_{j}\right)$.

Given its adjustable parameters, model 3 improves the fit, so that $R_{3}<R_{2}$. But is this reduction large enough for the null hypothesis of an unperturbed elliptical to be rejected? Because the null hypothesis is obtained by imposing two constraints,

$\Delta C_{j}=0, \quad \Delta S_{j}=0$ 
the required test is identical to that of LS. Thus, the probability that measurement errors of the unperturbed elliptical orbit could give $R \leq R_{3}$ for model 3 is

$p_{2}=\left(\frac{R_{3}}{R_{2}}\right)^{\beta_{3}}$

with $\beta_{3}=1 / 2\left(N-M_{3}\right)$, where $M_{3}=2$ is the number of parameters for the unconstrained model.

The probability $p_{2}$ determines the outcome of the first decision made about the reality of $e$. If $p_{2}>0.05$, the least-squares displacement vector $\boldsymbol{\delta}_{\mathbf{3}}$ is consistent with measurement errors. There is then no evidence for a perturbation, and so $e$ passes this first test of reality. This success implies that the amplitudes and phases of the first three harmonics of the velocity curve are consistent with Keplerian motion. But if $p_{2}<0.05$, a perturbation is detected, and so $e$ is suspect. Whether then to accept or reject $e$ is discussed further in Sect. 3.4.

\subsection{Detection of Keplerian harmonic}

Fitting Eq. (6) to the velocity curve tells us that its 3rd harmonic can be written as $\boldsymbol{H}_{\mathbf{3}}=\boldsymbol{K}_{\mathbf{3}}+\boldsymbol{\delta}_{\mathbf{3}}$, where $\boldsymbol{K}_{\mathbf{3}}=\left(C_{3}, S_{3}\right)$.

Independently of the significance of $\boldsymbol{\delta}_{\mathbf{3}}$ according to the $p_{2}$-test, we can ask if $\boldsymbol{K}_{\mathbf{3}}$ is detected. This harmonic is regarded as detected if the hypotheses that $\boldsymbol{K}_{\mathbf{3}}=\mathbf{0}$ and $\boldsymbol{H}_{\mathbf{3}}=\mathbf{0}$ are both rejected. The second hypothesis is necessary in order to reject solutions that imply a fortuitous near cancellation of $\boldsymbol{\delta}_{\mathbf{3}}$ and $\boldsymbol{K}_{\mathbf{3}}$.

To test the first hypothesis, $\boldsymbol{K}_{\mathbf{3}}$ is subtracted from the solution of Sect. 3.2 in order to see if it is essential to that fit's success. This subtraction is effected by again using Eq. (6) but now with constraints

$\Delta C_{j}=\delta C_{j}-C_{j}, \quad \Delta S_{j}=\delta S_{j}-S_{j}$.

The model thus constrained is not a physically realistic model of the velocity curve. Instead, it defines a null hypothesis set up as a "straw man" purely for testing the significance of $\boldsymbol{K}_{\boldsymbol{j}}$. Accordingly, if the "straw man" survives the test, we do not accept the null hypothesis but merely conclude that $\boldsymbol{K}_{\boldsymbol{j}}$ is too small to be detected.

With $\Delta_{j}$ fixed by Eq. (9), this model $(k=4)$ has no adjustable parameters, so that $R_{4}$ is derived arithmetically. Moreover, since Eq. (9) represents a displacement from the least-squares solution, $R_{4}>R_{3}$. But is this increase large enough to reject the null hypothesis? Because again two constraints have been imposed, the test is formally identical to that of Sect. 3.2. Thus, on the null hypothesis that model $k=4$ is the true model,

$q_{1}=\left(\frac{R_{3}}{R_{4}}\right)^{\beta_{3}}$

is the probability that measurement errors could give $R \leq R_{3}$ when model 3 is fitted to the data.

To test the second hypothesis, $\boldsymbol{H}_{3}$ is subtracted from the least-squares solution by again using Eq. (6) but now with constraints

$\Delta C_{j}=-C_{j}, \quad \Delta S_{j}=-S_{j}$.
With $\Delta_{j}$ thus fixed, this model $(k=5)$ also has no adjustable parameters, so that $R_{5}$ is derived arithmetically. Moreover, since Eq. (11) represents a displacement from the least-squares solution, $R_{5}>R_{3}$. But is this increase large enough to reject the hypothesis? Because again two constraints have been imposed, the test is again formally identical to that of Sect. 3.2. Thus, on the hypothesis that model 5 is the true model,

$q_{2}=\left(\frac{R_{3}}{R_{5}}\right)^{\beta_{3}}$

is the probability that measurement errors could give $R \leq R_{3}$ when model 3 is fitted to the data.

Given the outcomes of these two tests, we now define

$p_{3}=\max \left(q_{1}, q_{2}\right)$

which is such that $p_{3}<0.05$ implies $q_{1}<0.05$ and $q_{2}<0.05$. Accordingly, if $p_{3}<0.05, \boldsymbol{K}_{\mathbf{3}}$ is detected. But if $p_{3}>0.05, \boldsymbol{K}_{\mathbf{3}}$ is not regarded as detected, even if $q_{1}<0.05$.

\subsection{Decisions}

Following the $p_{1}$-test's detection of a significant $e$, its reality is subject to the $p_{2}$ - and $p_{3}$-tests. There are four possible outcomes:

\subsection{1. $p_{2}>0.05 ; p_{3}<0.05$}

The eccentricity of an $\mathrm{SB}$ in this domain of $\left(p_{2}, p_{3}\right)$-space is powerfully supported. Not only are the amplitudes and phases of the first three Keplerian harmonics consistent with the data, with no evidence of perturbation, but $\boldsymbol{K}_{\mathbf{3}}$ is detected. Thus the interpretation of the velocity curve's 2 nd harmonic $\boldsymbol{H}_{2}$ as due to orbital eccentricity is decisively confirmed. Follow-up investigations that require $e>0$ can be carried out with complete confidence.

\subsection{2. $p_{2}<0.05 ; p_{3}<0.05$}

The eccentricity of an SB in this domain has some support. Despite $\boldsymbol{\delta}_{\mathbf{3}}$ being significant, the detection of $\boldsymbol{K}_{\mathbf{3}}$ indicates an elliptical orbit. Nevertheless, the elements are uncertain because of the perturbation. Follow-up investigations that require $e>0$ are therefore risky because of the unknown systematic errors of $e$ and $\omega$.

\subsection{3. $p_{2}>0.05 ; p_{3}>0.05$}

The eccentricity of an SB in this domain is not strongly supported. Although $\boldsymbol{\delta}_{\mathbf{3}}$ is not significant, the failure to detect $\boldsymbol{K}_{\mathbf{3}}$ implies that $\boldsymbol{H}_{\mathbf{2}}$ may still be due to a 2 nd harmonic perturbation - see Sect. 4.1. Accordingly, follow-up investigations that require $e>0$ are subject to risk.

\subsection{4. $p_{2}<0.05 ; p_{3}>0.05$}

The eccentricity of an SB in this domain of $\left(p_{2}, p_{3}\right)$-space is in doubt. The amplitudes and phases of the first three Keplerian 
harmonics are inconsistent with the data, thus indicating a perturbed velocity curve. Moreover, the non-detection of $\boldsymbol{K}_{\mathbf{3}}$ undermines the interpretation of $\boldsymbol{H}_{\mathbf{2}}$ as due to an elliptical orbit. Follow-up investigations that require $e>0$ are inadvisable.

\subsection{Comments}

The following comments are intended to clarify the above tests:

a) If a circular orbit is not rejected by the $p_{1}$-test, its acceptance is recommended.

Some spectroscopists assume that the motivation for thus setting $e=0$ is a preference for simplicity; others believe that this decision is based only on statistical arguments. But LS (Sect. II) also stressed the physical argument "that the effect of tidal friction is to diminish the eccentricity of a binary system". Accordingly, when they discovered (LS, Sect. V) a high frequency of circular orbits for long-period SBs with giant components, they attributed such orbits to "energy dissipation in the deep convective envelopes of the giant component". A further discussion of the a posteriori evidence for the efficacy of tidal dissipation is given by Lucy \& Sweeney (1973).

When an observer finds that $e$ is not significant, he can claim, as did LS, to have discovered that a mechanism causing a secular decrease of $e$ has been operating. If so, it is unlikely that the secular decrease has been caught at the special epoch when $e \approx E(\hat{e})$, the expected value due to measurement errors when $e=0-$ Eq. (18) in LS. Accordingly, if $e$ is not significant, we expect that typically $e \ll E(\hat{e})$, and so the best option is to set $e=0$.

Of course, an observer may choose not to claim this discovery and so publish the elliptical orbit. But the resulting contamination of catalogues with $e$ 's that can be attributed to measurement errors is regrettable and misleading. A theorist interprets a small but non-zero $e$ as indicating a modest number of $e$-foldings in the secular decrease of $e$. But if $e$ is not significant, the data is consistent with an infinite number $e$-foldings.

The statistical and physical arguments for setting $e=0$ when $p_{1}>0.05$ were already compelling in the early 1970's, and they are even more so now.

b) If a circular orbit is accepted, its reality is not doubted. A perturbation that converts an elliptical orbit into an apparently circular one is possible but improbable.

c) Because $e$ is non-negative, errors in $e$ are non-Gaussian when $e \simeq 0$. In consequence, the estimate of $e$ derived from $e \cos \omega$ and $e \sin \omega$ has a positive bias, and this can mislead the investigator into concluding that $e$ is significant. To avoid such misjudgements, LS applied the statistical theory of hypothesis testing.

In this paper, the detectability of small, non-negative Fourier amplitudes is an issue. Again, the hypothesis-testing approach is preferable to comparing (biased) amplitudes to their standard errors.

d) Although $p_{1}<0.05$ is interpreted in Sect. 3.1 as the detection of a significant $e$, it could more accurately be described as the detection of a significant $\boldsymbol{H}_{2}$ - see Sect. 2.2. Since perturbations by gas streams or proximity effects can introduce a 2nd harmonic even when $e=0$, the detection of $\boldsymbol{H}_{\mathbf{2}}$ cannot of itself be decisive evidence for an elliptical orbit. e) The statistical tests of Sects. 3.1-3.3 assume normallydistributed measurement errors and models that are linear in the adjustable parameters. The required linearity holds rigorously for the $p_{2}$ - and $p_{3}$-tests - see Eq. (6) - but not for the $p_{1}$-test. For this latter test, Monte Carlo experiments (Lucy 1989) confirm its validity.

f) The adjustable 3rd harmonic in Eq. (6) is not a model of the perturbation. We assume only that the perturbation has period $P$ and has a Fourier expansion with non-zero amplitudes for one or more of the first three harmonics. In particular, even though we seek evidence of a perturbation via the third harmonic, the efficacy of the $p_{2}$-test does not require the perturbation to contain this harmonic.

To illustrate this point, let us consider an error-free velocity curve subject to the 1 st harmonic perturbation $\epsilon K \cos L$. The resulting modified elements, denoted by primes, are derived using the leading-terms formulae from Sect. 2.2. Since only the cosine term of the 1 st harmonic is perturbed, we have $\tilde{C}_{1}=(1+\epsilon) C_{1}=(1+\epsilon) K$, with all other estimated coefficients having their exact values. Accordingly, we find $\gamma^{\prime}=\gamma$, $K^{\prime}=K(1+\epsilon), \omega^{\prime}=\omega$, and $e^{\prime}=e /(1+\epsilon)$. On the basis of these elements, we predict $C_{3}^{\prime}=C_{3} /(1+\epsilon)$ and similarly for $S_{3}^{\prime}$. Thus, as anticipated in Sect. 2.3, there is a discrepancy between the predicted vector $\left(C_{3}^{\prime}, S_{3}^{\prime}\right)=\left(C_{3}, S_{3}\right) /(1+\epsilon)$ and its estimate $\left(\tilde{C_{3}}, \tilde{S_{3}}\right)=\left(C_{3}, S_{3}\right)$.

g) When the $p_{2}$-test detects a perturbation, the orbital elements are subject to unkown systematic errors. No statistical procedure can then extract unbiased elements from the velocity curve. Additional data or a physical model of the perturbation is required to derive more reliable elements.

h) Equation (13) in LS defines a quantity

$\mu=\frac{\sigma}{K} \sqrt{\frac{2}{N}}$

which is a dimensionless measure of a velocity curve's precision. In terms of $\mu, e$ should be detectable at the 5 per cent level if

$e>2.45 \mu$

- see Eq. (22) in LS.

An essentially identical analysis allows criteria to be derived for the detection of Keplerian harmonics in perturbationfree orbits with $e^{2} \ll 1$. Thus, the 5 per cent criteria are

$e^{2}>2.18 \mu$

for $\boldsymbol{K}_{\mathbf{3}}$, and

$e^{3}>1.84 \mu$

for $\boldsymbol{K}_{\mathbf{4}}$. Generally, $\boldsymbol{K}_{\boldsymbol{j}}$ should be detectable at the $100 p$ per cent level of significance if

$e^{j-1}>\frac{\sqrt{-2 \ln p}}{a_{j}} \mu$

where $a_{j}$ is given by Eq. (A.11).

The inequality given by Eq. (16) defines the domain in $(e, \mu)$-space within which $e$ can be confirmed by the $p_{3}$-test. 
Ideally, an observing campaign should be designed to obtain a velocity curve that falls in this domain.

i) The $p_{2}$-test assumes that the perturbation has period $P$. But some causes of perturbation violate this assumption. One example is pulsations, another is spots, and yet another is a third-body perturbation. In general, such effects increase the residuals and hence the deduced measurement error $\sigma$ and are not detected by $p_{2}$.

j) The $p_{2}$-test relies entirely on radial velocities for detecting a perturbation. Phase-dependent line strengths and line shapes may provide independent evidence of perturbation. Such evidence may also resolve the ambiguity noted in Sect. 3.4.3.

k) Following common practice, we have adopted the 5 per cent level of significance in stating the decisions reached from the values of $p_{1,2,3}$. But the level can be varied. In particular, the impact of erroneous conclusions on subsequent investigations should be considered.

1) Many astronomers are now familiar with defining confidence intervals about a minimum- $\chi^{2}$ solution in terms of an increment $\Delta \chi^{2}-$ e.g. Press et al. (1992). The relevant statistical theory is related to that from which the tests of Sect. 3 are derived.

In the limit $N \rightarrow \infty$, the tests of Sect. 3 simplify to

$\Delta \chi^{2}=-2 \ln p$

so that $p<0.05$ corresponds to $\Delta \chi^{2}>5.99$. Equation (19) reproduces the column for $v=2$ in the table on p. 692 of Press et al. (1992), except that their $p$ is the enclosed, and ours the excluded, probability.

Note that, in contrast to Eq. (19), the tests of Sect. 3 do not require $N \rightarrow \infty$. Monte Carlo simulations confirm that they remain valid even when $N$ only slightly exceeds $M$.

\section{Monte Carlo experiments}

In this section, the tests of Sect. 3 are applied to synthetic velocity curves. With the exact elements known, these experiments investigate the reliability of the statistical tests.

\subsection{Perturbed circular orbit}

Probably the commonest error for SBs is the assignment of a significant $e$ to a binary with vanishingly small $e$ but subject to a 2 nd harmonic perturbation. To simulate this case, synthetic data sets $\left(v_{n}, t_{n}\right)$ are generated from the formula

$v_{n}=V_{n}+\epsilon K \cos \left(2 L_{n}-\varpi\right)+\sigma z_{n}$.

Here the first term $V_{n}$ is the exact radial velocity at $t_{n}$ for a circular orbit of semi-amplitude $K$; the second term is a 2 nd harmonic perturbation of dimensionless amplitude $\epsilon$; and the third term is a random Gaussian measurement error with variance $\sigma^{2}$.

A least-squares elliptical orbit for such a velocity curve will have a spurious $e \approx \epsilon$ with $\omega \approx \varpi$. By repeated sampling, we can investigate the success of the $p_{2}$ - and $p_{3}$-tests in detecting these spurious $e$ 's despite their possible significance according to the $p_{1}$-test.

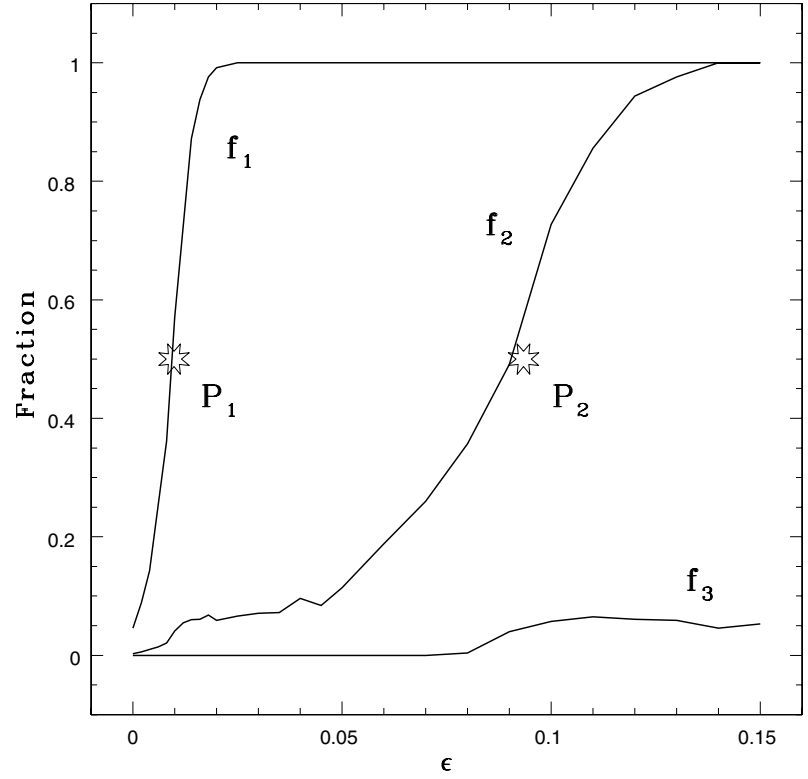

Fig. 1. Monte Carlo simulations. The fractions $f_{1,2,3}$ of synthetic velocity curves with $p_{1,2,3}<0.05$ are plotted against $\epsilon$, the fractional amplitude of the 2 nd harmonic perturbation in Eq. (20). The starred points indicate the theoretical values of $\epsilon$ at which typically the spurious $e$ becomes significant $\left(P_{1}\right)$ and the perturbation is detected $\left(P_{2}\right)$.

The first experiment is as follows. For fixed $\epsilon$, we generate and analyse $10^{3}$ synthetic velocity curves with random $\varpi$ 's and uniformly-distributed $L_{n}$. Each data set comprises $N=50$ velocities $v_{n}$ with $\sigma / K=0.02$, so that $\mu=4 \times 10^{-3}$. Finally, the least-squares orbits assume $P$ fixed at its exact value. From the $10^{3}$ analyses, the fractions $f_{1,2,3}$ with $p_{1,2,3}<0.05$ are computed, and this is then repeated with $\epsilon$ increasing from 0 to 0.15 .

The results are plotted in Fig. 1 . The $f_{1}$-curve shows that significant $e$ 's increase sharply from $\approx 5$ per cent at $\epsilon=0$ to 100 per cent for $\epsilon \gtrsim 0.02$. The 50 per cent detection rate occurs close to the value $\epsilon_{1}=2.45 \mu=0.0098$ expected heuristically from Eq. (15) - the point $\mathrm{P}_{1}$. The $f_{2}$-curve shows that for $\epsilon \lesssim$ 0.06 only a small fraction of the significant $e$ 's are detected as spurious by the $p_{2}$-test. But with further increase of $\epsilon$, the absence of $\boldsymbol{K}_{\mathbf{3}}$ in Eq. (20) becomes detectable, and the $f_{2}$-curve rises to give 100 per cent detections of $\delta_{3}$ when $\epsilon \gtrsim 0.12$. The 50 per cent rate occurs close to the value $\epsilon_{2}=0.093$ similarly expected from Eq. (16) - the point $\mathrm{P}_{2}$.

Finally, the $f_{3}$-curve shows that for $\epsilon \gtrsim 0.09$, a small fraction $(\approx 0.06)$ of spurious $e$ 's are adjudged real by the $p_{3}$-test.

This experiment demonstrates that, for perturbed circular orbits, the tests of Sect. 3 perform as expected. But the results also emphasize that if a significant $e$ is such that

$2.45 \mu \lesssim e \lesssim \sqrt{2.18 \mu}$

then we cannot decide if $e$ is real or spurious. In this interval, the $p_{2}$-test cannot detect a spurious $e$, and the $p_{3}$-test fails to confirm a real $e$.

\subsection{Perturbed elliptical orbit}

In a second experiment, $e=0$ in Eq. (20) is replaced by $e=$ 0.12 with random $\omega$. Other details are unchanged. 
From a sequence of simulations with $\epsilon$ increasing from 0 to 0.15 , we find that the $p_{2}$-test detects the perturbation with a reliability of 50 and 90 per cent at $\epsilon \approx 0.035$ and 0.06 , respectively. The $p_{3}$-test confirms the reality of $e$ with a reliabilty that drops from $\approx 95$ per cent at $\epsilon=0$ to $\approx 60$ per cent at $\epsilon=0.15$. This drop when $\epsilon \approx e$ is not surprising since the imposed perturbation in the 2nd harmonic then has an amplitude $\approx\left|\boldsymbol{K}_{\mathbf{2}}\right|$.

\subsection{Unperturbed elliptical orbits}

In a third experiment, the perturbation amplitude $\epsilon=0$ and $e$ is now the varied parameter.

From a sequence of simulations with $e$ increasing from 0 to 0.13 , we find, as expected from Sect. $3.5 \mathrm{i}$ ), that the $p_{1}$-test has a $\gtrsim 50$ per cent succes rate in detecting $e$ when $e \gtrsim 2.45 \mu$, and the $p_{3}$-test a $\gtrsim 50$ per cent succes rate in confirming the reality of $e$ when $e^{2} \gtrsim 2.18 \mu$.

\section{Examples}

In this section, the tests developed in Sect. 3 are illustrated by applying them to SBs with published orbits.

\subsection{HD 45088}

This SB is the subject of the first paper (Griffin \& Emerson 1975 ) in Griffin's series on spectroscopic orbits from photoelectric radial velocities. The authors report a high quality orbit with $e=0.150 \pm 0.004$ derived from $N=54$ measured velocities with standard error $\sigma=1.0 \mathrm{~km} \mathrm{~s}^{-1}$. The eccentricity is clearly highly significant but, given the relatively short period of 6.992 , one might suspect that the orbit is circular and $e$ spurious.

The first step is to apply the LS-test for the significance of $e$. This and subsequent calculations are carried out with $P$ fixed at the value determined by the original authors, so that $M_{2}=5$. The result of the LS-test is $p_{1}=5.6 \times 10^{-38}$, confirming the overwhelming significance of $e$.

The second step is to compare this elliptical orbit to the fit obtained with Eq. (6) when $j=3$. The least-squares fit gives $\delta C_{3}=0.28 \pm 0.20 \mathrm{~km} \mathrm{~s}^{-1}$ and $\delta S_{3}=0.07 \pm 0.19 \mathrm{~km} \mathrm{~s}^{-1}$, suggesting little or no evidence for $\boldsymbol{\delta}_{\mathbf{3}}$ beyond what can be attributed to measurement errors. This is confirmed by Eq. (8), which gives $p_{2}=0.35$. The amplitudes and phases of the first three harmonics of the velocity curve of HD 45088 are therefore consistent with unperturbed Keplerian motion.

The elliptical orbit of HD 45088 is now subject to the test of Sect. 3.3. The 3rd harmonic coefficients computed from the least-squares elliptical orbit are $C_{3}=-1.26 \mathrm{~km} \mathrm{~s}^{-1}$ and $S_{3}=$ $0.58 \mathrm{~km} \mathrm{~s}^{-1}$. Subtracting this component according to Eqs. (6) and (9), then computing $R_{4}$, we find that $q_{1}=1.2 \times 10^{-7}$. Then, similarly subtracting $\boldsymbol{H}_{\mathbf{3}}$ according to Eqs. (6) and (11), we find that $q_{2}=3.9 \times 10^{-6}$. Accordingly, $p_{3}=q_{2}=3.9 \times 10^{-6}$ and so there is a highly significant detection of $\boldsymbol{K}_{\mathbf{3}}$. This confirmation of ellipticity and the earlier non-detection of perturbation place HD 45088 in the domain of $\left(p_{2}, p_{3}\right)$-space containing orbits with the most reliable $e$ 's.

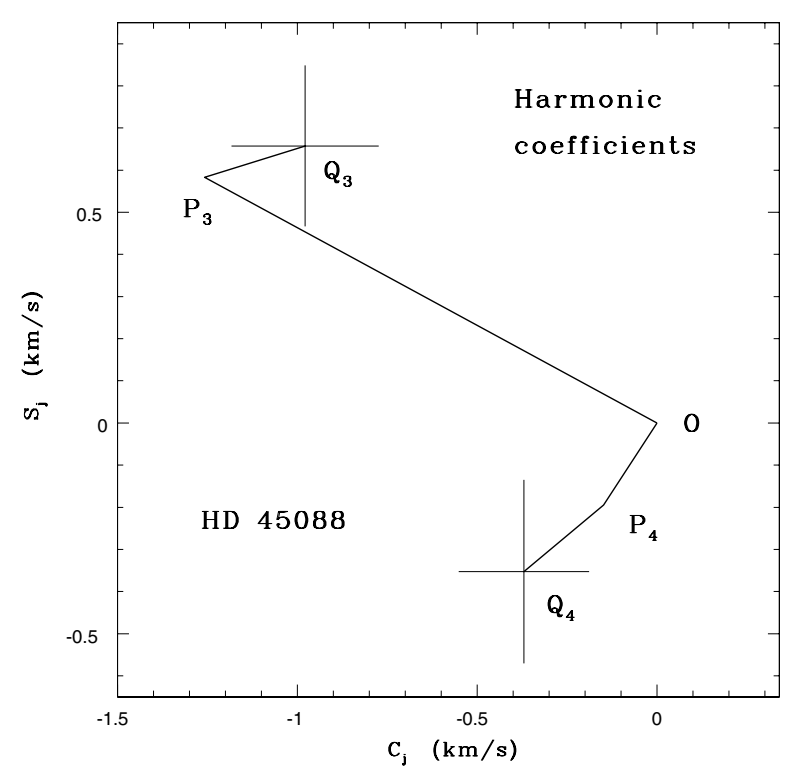

Fig. 2. Fourier coefficients for HD 45088. The 3rd and 4th harmonics of the least-squares elliptical orbit are shown as the vectors $\boldsymbol{O P}_{\mathbf{3}}$ and $\boldsymbol{O} \boldsymbol{P}_{\mathbf{4}}$, respectively. The displacement vectors $\boldsymbol{\delta}_{\mathbf{3}, \mathbf{4}}$ obtained by fitting Eq. (6) are also plotted together with their $1 \sigma$ error bars.

Having detected $\boldsymbol{K}_{\mathbf{3}}$, we can extend the search to $\boldsymbol{K}_{\mathbf{4}}$. However, the predicted coefficients $C_{4}=-0.15 \mathrm{~km} \mathrm{~s}^{-1}$ and $S_{4}=-0.19 \mathrm{~km} \mathrm{~s}^{-1}$ are somewhat too small for detection.

The calculations for the 3rd and 4th harmonics are illustrated in Fig. 2. For the 3rd harmonic, we see that the components of $\boldsymbol{\delta}_{\mathbf{3}} \equiv \boldsymbol{P}_{\mathbf{3}} \boldsymbol{Q}_{\mathbf{3}}$ are comparable with their standard errors, in agreement with their non-significance according to $p_{2}$. It is also evident that $\boldsymbol{K}_{\mathbf{3}} \equiv \boldsymbol{O P}_{\mathbf{3}}$ is the dominant contribution to $O Q_{3}$, so that its subtraction significantly increases the residuals, leading to its highly significant detection $\left(p_{3} \ll 0.05\right)$.

The corresponding vectors and error bars for the 4th harmonic are also plotted in Fig. 2. These show that detection of this harmonic is just beyond reach.

The outcome of this investigation of HD 45088 is that it passes all tests that can be usefully carried out given the precision of the Griffin-Emerson velocity curve. Needless to say, new data with $\mu$ reduced significantly below the GriffinEmerson value of $3.7 \times 10^{-3}$ might reveal a perturbation that is currently undetectable. On the other hand, if a perturbation were still not found, the confirmation of Keplerian motion could be extended to the 4 th and higher harmonics.

For the above value of $\mu$, Eq. (15) predicts that $e>0.009$ is required for the detection of $e$. This is consistent with $e=$ 0.15 being overwhelmingly significant according to the $p_{1}$-test. Similarly, Eq. (16) predicts that $e>0.09$ is required for the detection of $\boldsymbol{K}_{\mathbf{3}}$, consistent with its highly significant detection according the $p_{2}$-test. Finally, Eq. (17) predicts that $e>0.19$ is required for the detection of $\boldsymbol{K}_{\mathbf{4}}$, consistent with its non-detection.

\section{2. $\zeta$ Trianguli Australis}

For this 12d.976 SB, Skuljan et al. (2004) report 225 radial velocities obtained with a high-resolution fibre-fed spectrograph. As noted in Sect. 1, the gain in precision over the earlier 


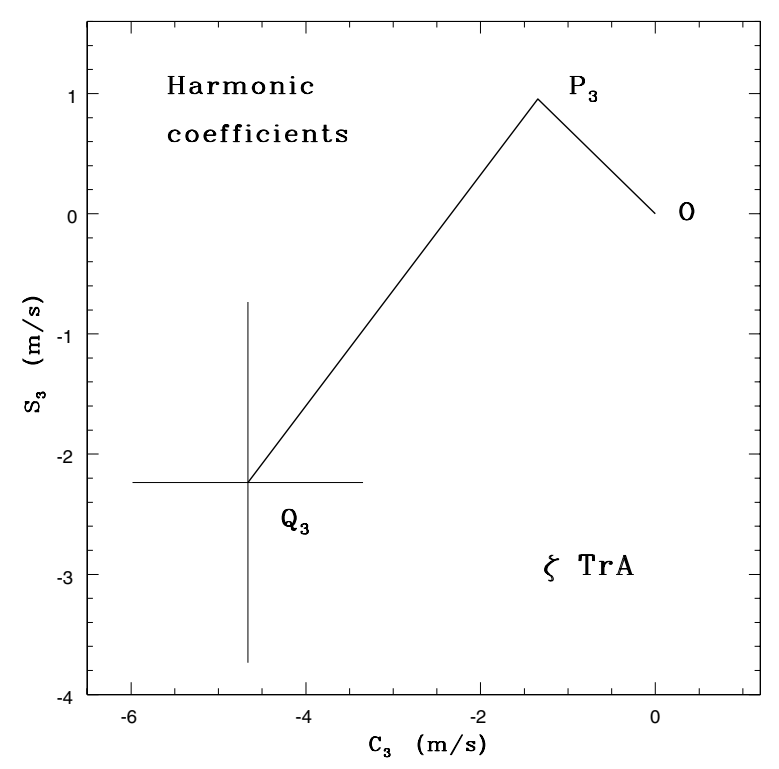

Fig. 3. Fourier coefficients for $\zeta \operatorname{TrA}$. The 3rd harmonic of the leastsquares elliptical orbit is shown as the vector $\boldsymbol{O P}_{\mathbf{3}}$. The displacement vector $\delta_{3}$ obtained by fitting Eq. (6) is also plotted as $\boldsymbol{P}_{\mathbf{3}} \boldsymbol{Q}_{\mathbf{3}}$ together with $1 \sigma$ error bars.

orbit by Spencer Jones (1928) is a remarkable two orders of magnitude.

Recomputing the orbit with $P$ fixed (their Table 6), we find $p_{1}=1.5 \times 10^{-148}$, in agreement with their claim of high statistical significance despite the small eccentricity of 0.014 .

Let us now investigate the reality of $e$ by applying the tests of Sects. 3.2 and 3.3. The first step is to fit Eq. (6) to their data. Switching the unit of velocity to metres per s, we obtain $\delta C_{3}=-3.3 \pm 1.3 \mathrm{~m} \mathrm{~s}^{-1}$ and $\delta S_{3}=-3.2 \pm 1.5 \mathrm{~m} \mathrm{~s}^{-1}$, which suggest a significant perturbation. This is confirmed by finding $p_{2}=0.0063$. Accordingly, the null hypothesis of an unperturbed elliptical orbit is rejected.

The next step is to seek confirmation of $\boldsymbol{K}_{\mathbf{3}}$, for which the predicted components are $C_{3}=-1.34 \mathrm{~m} \mathrm{~s}^{-1}$ and $S_{3}=$ $0.96 \mathrm{~m} \mathrm{~s}^{-1}$. Since these are comparable to the standard errors on the components of $\boldsymbol{\delta}_{\mathbf{3}}$, we immediately suspect that there is little support for the detection of this harmonic. This is confirmed by the $p_{3}$-test. Subtracting $\boldsymbol{K}_{\mathbf{3}}$, we find that $q_{1}=0.47$. Similarly, subtracting $\boldsymbol{H}_{3}$, we find that $q_{2}=9.9 \times 10^{-4}$. Accordingly, $p_{3}=q_{1}=0.47$, and so $\boldsymbol{K}_{\mathbf{3}}$ is not detected.

These results place $\zeta \operatorname{TrA}$ in the domain of $\left(p_{2}, p_{3}\right)$-space discussed in Sect. 3.4.4. This contains the least reliable category of $e$ 's. The dramatically improved precision has allowed the discovery of a very small amplitude yet highly significant $\boldsymbol{H}_{2}$. But its interpretion as due to orbital eccentricity is put in doubt by the detection of a significant $\boldsymbol{\delta}_{\mathbf{3}}$ and the nondetection of $\boldsymbol{K}_{\mathbf{3}}$. The amplitude of $\boldsymbol{H}_{\mathbf{2}} \sim 105 \mathrm{~m} \mathrm{~s}^{-1}$ is so small that there is no difficulty in attributing it entirely to the detected perturbation.

The above calculations are illustrated in Fig. 3. Comparison with Fig. 2 for HD 45088 is illuminating. For HD 45088, $\boldsymbol{O Q} Q_{3} \equiv \boldsymbol{H}_{3}$ is dominated by $\boldsymbol{O P _ { 3 }} \equiv \boldsymbol{K}_{3}$. In contrast, for $\zeta \operatorname{TrA}$, $\boldsymbol{O} Q_{3}$ is dominated by $\boldsymbol{P}_{\mathbf{3}} \boldsymbol{Q}_{\mathbf{3}} \equiv \boldsymbol{\delta}_{\mathbf{3}}$. This difference accounts for detection of $\boldsymbol{K}_{\mathbf{3}}$ for HD 45088 and its non-detection for $\zeta \operatorname{TrA}$.
Despite the enormous statistical significance of the $e$ reported by Skuljan et al. (2004), it fails to pass the tests of reality developed in Sect. 3.2 and 3.3. Thus follow-up investigations predicated on the reality of $e$ carry substantial risk of misleading or erroneous conclusions. Nevertheless, just as $p_{1}>0.05$ does not prove that an orbit is not eccentric but merely that $e$ is not significant, so a finding that $p_{2}<0.05$ and $p_{3}>0.05$ does not prove that $e$ is not real but merely that this supposition does not find significant support in the data.

\section{Conclusion}

The aim of this paper has been to develop tests that allow SBs with elliptical orbits of high reliability to be separated from those with spurious $e$ 's. The example of HD 45088 shows that the literature contains orbits that are not challenged by these tests. But $\zeta \operatorname{Tr} A$ shows that some published $e$ 's are, as expected, probably due to perturbations. Evidently, extensive further recomputations would greatly clarify the reliability of the accumulated data on SBs. Indeed, categorizing SBs according to their location in the $\left(p_{1}, p_{2}, p_{3}\right)$-cube offers an objective treatment of orbit quality that could replace or complement the subjective judgements (e.g., $a, b, c, \ldots$ ) traditionally used by compilers of catalogues. Note that the recently published 9th catalogue of SB orbits (Pourbaix et al. 2004) compiles individual velocities, thus greatly facilitating orbit recomputations.

As to measuring apsidal motion with high precision velocity curves (Sect. 1), observers should select SBs with $p_{1}<0.05$ and $p_{2}>0.05$, and then aim for a new orbit that satisfies the first and if possible also the second of the inequalities given in Eqs. (16) and (17). By doing so, tests for the detection of $\boldsymbol{K}_{\mathbf{3}}$ and possibly also $\boldsymbol{K}_{\mathbf{4}}$ can be carried out. Such detections would establish the reality of $e$ and thus justify 2nd epoch observations.

In contrast, re-observing systems with $p_{1}>0.05$ in the hope of discovering hitherto undetected $e$ 's runs the following risks: that $e$ remains undetectable; that $e$ is significant but a perturbation is detected; that $e$ is significant and no perturbation is detected but $e$ is too small for detection of $\boldsymbol{K}_{\mathbf{3}}$. Evidently, this (re-)observing strategy will have a much smaller discovery rate of orbits in the highest category of reliability (Sect. 3.4.1).

A long standing problem for orbits of SBs is their nonuniform distribution of $\omega$, sometimes referred to as the Barr effect (see, e.g., Batten \& Ovenden 1968). This was present in the orbits published by LS, both for those with and those without significant $e$ 's, and was attributed to perturbations. Accordingly, it will be of interest to re-examine the distribution of $\omega$ for orbits with and without perturbations according to the $p_{2}$-test. An obvious prediction is that orbits with $p_{2}>0.05$ will have a negligible or much reduced Barr effect, and those with $p_{2}<0.05$ will have the effect enhanced.

Given the typical precision of published velocity curves, a programme of recomputation would investigate the effects of perturbations with amplitudes $\gtrsim 0.1 \mathrm{~km} \mathrm{~s}^{-1}$. But the work of Skuljan et al. (2004) demonstrates that perturbations are detectable to much lower amplitudes. In this regard, it would be interesting to observe eclipsing binaries with light curves that imply $e \cos \omega=0$ to high precision. Comparison with the 
spectroscopic values of $e \cos \omega$ could then reveal, or demonstrate the absence of, perturbations down to $\sim 1 \mathrm{~m} \mathrm{~s}^{-1}$.

\section{Appendix A: Fourier coefficients}

The Keplerian velocity of a binary component can be expanded in a Fourier series in the mean anomaly $M$ (e.g., Plummer 1908). This is readily transformed into the Fourier series in the mean longitude $L=M+\omega$ given in Eq. (2). If we define $\alpha=K\left(1-e^{2}\right) \cos \omega$ and $\beta=K \sqrt{1-e^{2}} \sin \omega$, the Fourier coefficients are

$C_{j}=\alpha c_{j} \cos j \omega+\beta s_{j} \sin j \omega$

and

$S_{j}=\alpha c_{j} \sin j \omega-\beta s_{j} \cos j \omega$.

Expressions for the coefficients $c_{j}$ and $s_{j}$ in terms of Bessel functions are given by Plummer (1908). Expansions of the latter as power series in $e$ (e.g., Smart 1953, p. 378) yields the following formulae, accurate to $O\left(e^{7}\right)$

$$
\begin{aligned}
& c_{1}=1-\frac{1}{8} e^{2}+\frac{1}{192} e^{4}-\frac{1}{9216} e^{6} \\
& s_{1}=1-\frac{3}{8} e^{2}+\frac{5}{192} e^{4}-\frac{7}{9216} e^{6} \\
& c_{2}=e\left[1-\frac{1}{3} e^{2}+\frac{1}{24} e^{4}-\frac{1}{360} e^{6}\right] \\
& s_{2}=e\left[1-\frac{2}{3} e^{2}+\frac{1}{8} e^{4}-\frac{1}{90} e^{6}\right] \\
& c_{3}=a_{3} e^{2}\left[1-\frac{9}{16} e^{2}+\frac{81}{640} e^{4}\right]
\end{aligned}
$$

$$
\begin{aligned}
& s_{3}=a_{3} e^{2}\left[1-\frac{15}{16} e^{2}+\frac{189}{640} e^{4}\right] \\
& c_{4}=a_{4} e^{3}\left[1-\frac{4}{5} e^{2}+\frac{4}{15} e^{4}\right]
\end{aligned}
$$

$$
s_{4}=a_{4} e^{3}\left[1-\frac{6}{5} e^{2}+\frac{8}{5} e^{4}\right]
$$

where

$a_{j}=\frac{1}{(j-1) !}\left(\frac{j}{2}\right)^{j-1}$.

Values of $C_{j}$ and $S_{j}$ obtained with these expansions have been checked against values obtained by numerical integration of the Fourier integrals.

\section{References}

Batten, A. H., \& Ovenden, M. W. 1968, PASP, 80, 85 Griffin, R. F., \& Emerson, B. 1975, Observatory, 95, 23 Lucy, L. B. 1989, Observatory, 109, 100

Lucy, L. B., \& Sweeney, M. A. 1971, AJ, 76, 544

Lucy, L. B., \& Sweeney, M. A. 1973, Observatory, 93, 37

Monet, D. G. 1979, ApJ, 234, 275

Plummer, H. C. 1908, ApJ, 28, 212

Pourbaix, D., Tokovinin, A. A., Batten, A. H., et al. 2004, A\&A, 424, 727

Press, W. H., Teukolsky, S. A., Vetterling, W. T., \& Flannery, B. P. 1992, Numerical Recipes (Cambridge: Cambridge Univ. Press) Russell, H. N. 1902, ApJ, 15, 252

Skuljan, J., Ramm, D. J., \& Hearnshaw, J. B. 2004, MNRAS, 352, 975

Smart, W. M. 1953, Celestial Mechanics (London: Longmans Green), 378

Wilsing, J. 1893, AN, 134, 90 